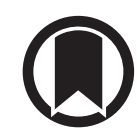

CrossMark

\title{
The DisEntangling Chronic Obstructive pulmonary Disease Exacerbations clinical trials NETwork (DECODE-NET): rationale and vision
}

\author{
Alexander G. Mathioudakis (10 ${ }^{1,2}$, Pradeesh Sivapalan ${ }^{3,4}$, Alberto Papi $\mathbb{C}^{5}$ and \\ Jørgen Vestbo (1) ${ }^{1,2}$ on behalf of the DECODE-NET (DisEntangling Chronic \\ Obstructive pulmonary Disease Exacerbations clinical trials NETwork) \\ Investigators 6
}

Affiliations: ${ }^{1}$ Division of Infection, Immunity and Respiratory Medicine, School of Biological Sciences, The University of Manchester, Manchester Academic Health Science Centre, Manchester, UK. ${ }^{2}$ The North West Lung Centre, Wythenshawe Hospital, Manchester University NHS Foundation Trust, Manchester, UK. ${ }^{3}$ Section of Respiratory Medicine, Dept of Internal Medicine, Herlev-Gentofte Hospital, Hellerup, Denmark. ${ }^{4}$ Dept of Internal Medicine, Zealand University Hospital, Roskilde, Denmark. ${ }^{5}$ Research Center on Asthma and COPD, Dept of Medical Sciences, University of Ferrara, Ferrara, Italy. ${ }^{6} \mathrm{~A}$ list of the DECODE-NET Investigators can be found in the acknowledgements section.

Correspondence: Jørgen Vestbo, Division of Infection, Immunity and Respiratory Medicine, School of Biological Sciences, The University of Manchester, UK. E-mail: jorgen.vestbodmanchester.ac.uk

@ERSpublications

The DisEntangling Chronic Obstructive pulmonary Disease Exacerbations clinical trials NETwork: \#DECODE_NET. Vision statement of an emerging, global network aiming to conduct collaborative clinical research on COPD exacerbation management @ERSpublications https://bit.ly/36kISgp

Cite this article as: Mathioudakis AG, Sivapalan P, Papi A, et al. The DisEntangling Chronic Obstructive pulmonary Disease Exacerbations clinical trials NETwork (DECODE-NET): rationale and vision. Eur Respir J 2020; 56: 2000627 [https://doi.org/10.1183/13993003.00627-2020].

\section{Introduction}

Acute exacerbations are largely responsible for the poor outcomes characterising COPD, the third leading cause of death globally and associated with a significant disability and socioeconomic burden $[1,2]$. Every year, $22-40 \%$ of all people suffering from COPD experience at least one moderate or severe exacerbation, while $9-16 \%$ experience more than one [3, 4]. Exacerbations are associated with an increased risk of subsequent exacerbations, cardiac events, worse quality of life and an accelerated lung function decline [5]. $15 \%$ of all severe exacerbations requiring hospitalisation lead to death within 90 days [6]. Thus, it is not surprising that patients consider exacerbations and hospitalisations due to exacerbations the most important, burdensome outcomes of COPD [7].

In contrast to the rapidly evolving management of other diseases, such as acute coronary syndrome, the management of exacerbations remains insufficient, with little advancement for decades; they thus represent a major, unaddressed global health need $[1,2]$. To improve the management and outcomes of COPD exacerbations, we launched an international, multidisciplinary clinical research network, the DECODE-NET (DisEntangling Chronic Obstructive pulmonary Disease Exacerbations: The DECODE-NET clinical trials NETwork), aiming to identify and remedy potential issues delaying the 
development of novel therapeutic approaches, and to conduct high-quality collaborative clinical research on the management of COPD exacerbations, with a focus on clinical trials. Here, we summarise crucial gaps in our clinical knowledge as well as the methodological challenges of conducting trials on the management of COPD exacerbations and how the DECODE-NET aspires to address them.

\section{Knowledge gaps complicating clinical research on COPD exacerbations}

Clinical research is complicated by our limited understanding of exacerbations and their underlying mechanisms. Firstly, COPD exacerbations are still loosely defined as acute episodes characterised by a deterioration in the respiratory symptoms, in patients with pre-existing COPD [8]. In the absence of accurate diagnostic biomarkers, diagnosis is solely based on clinical presentation $[9,10]$. Sensitivity and specificity of this case definition is limited by the significant day-to-day symptom variability in stable COPD, and the variability in symptom perception and reporting by patients $[2,11]$. As a result, some of the events currently approached as exacerbations in fact represent symptom variability in patients with stable COPD, not associated with short- or long-term adverse outcomes. Furthermore, the diagnostic criteria of a COPD exacerbation are also fulfilled by other respiratory or non-respiratory presentations (e.g. cardiovascular diseases) [12]. Inclusion of such events in clinical research studies introduces nondifferential misclassification bias, thereby reducing the strength of those studies. Moreover, assessment of the severity of exacerbation is often insufficient, most often relying on clinicians' decision to administer antibiotics and/or systematic corticosteroids (moderate) or to admit patients to the hospital (severe exacerbations) [1, 2]. However, in the absence of prognostic biomarkers these assessments are arbitrary and inconsistent. Consequently, there is a need to develop accurate diagnostic and prognostic biomarkers to identify those events associated with adverse short- and long-term sequelae, in the same way that troponin can differentiate a myocardial infarction from an inconsequential episode of angina in patients with ischaemic heart disease [13].

Exacerbations are heterogeneous, with different phenotypes, reflecting diverse aetiologies and underlying mechanisms that require a personalised treatment approach (figure 1) [14]. However, in the absence of cost-effective, accurate biomarkers able to provide timely clinical characterisation, exacerbations are still approached as a single disease entity, both in clinical practice, and also in most research studies. This could cause "dilution" of potential clinical impact of treatments that are effective only in a subgroup of exacerbations. This is probably the reason why antibiotics and systemic corticosteroids were only found to have modest effects in unselected exacerbations [15, 16]. Indeed, studies using emerging therapeutic biomarkers confirm that the impact of these treatments is limited to specific subgroups [17-19]. Shortcomings in clinical characterisation of exacerbations have delayed the introduction of precision medicine interventions in the management of exacerbations [20].

\section{Challenges in the design and conduct of clinical trials on COPD exacerbations}

The acute nature of COPD exacerbations complicates the conduct of clinical research studies evaluating their management [21-24]. Events qualifying for recruitment cannot be planned and often happen at inconvenient times. Potential participants are acutely unwell patients who experience severe respiratory symptoms and stress and are, therefore, less prepared (or capable) to receive information about a research study or consent to participate. Moreover, numerous clinicians are involved in the management of inpatient participants and need to be aware of the ongoing trial, prepared to follow the trial procedures and to adhere to the interventions. Concomitant treatments are difficult to standardise. As a result, such trials are very expensive, and require specific expertise, infrastructure and large research teams. However, our colleagues in cardiology, for example, have overcome this hurdle, and in DECODE-NET we believe this is an area worthy of such commitment of resources.

The design of clinical research studies, and specifically randomised controlled trials (RCTs), on the management of COPD exacerbations is also complex. Firstly, the heterogeneity of COPD exacerbations results in a need for more complex clinical trial designs, such as precision medicine trials or adaptive trial designs [25]. Moreover, the previously mentioned barriers of diagnostic inaccuracy, disease heterogeneity and lack of accurate therapeutic biomarkers complicates the selection of eligibility criteria that would allow for the recruitment of a homogeneous population likely to respond to an experimental treatment [11].

Selection of study endpoints is also challenging. Clinical research studies need to evaluate outcome measures that are important to patients, using standardised outcome definitions to facilitate comparing and synthesising the results of different studies [26]. In cases of studies evaluating acute events, such as COPD exacerbations, timing of the evaluation of outcomes is also crucial, as use of different timepoints could yield different results. A methodological survey revealed that trials of management of COPD exacerbations conducted during the past decade frequently have significant methodological limitations [27]. Characteristically, rates of treatment success or failure were only evaluated in $63 \%$ of all RCTs, and 


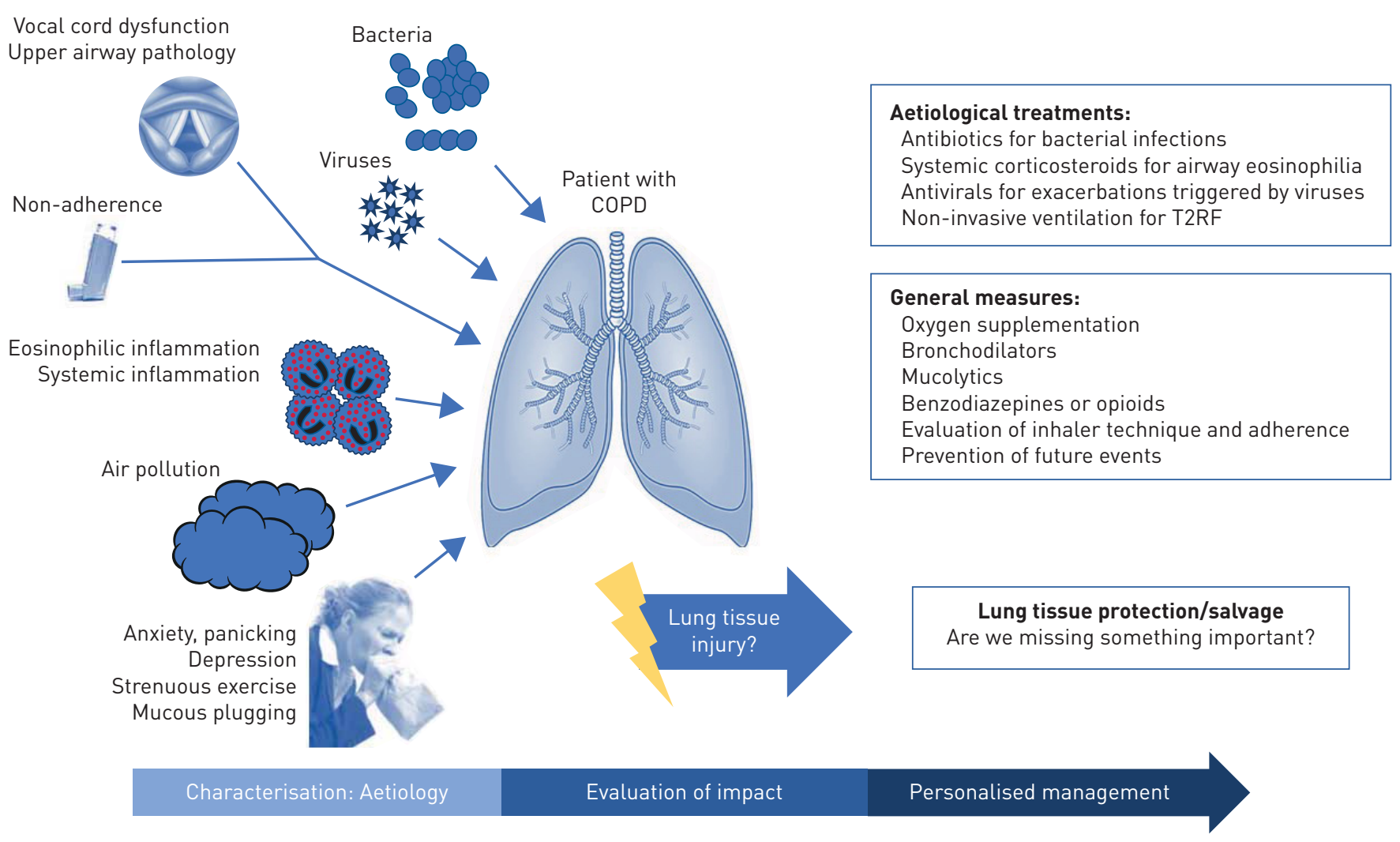

FIGURE 1 Characterisation and targeted treatments of COPD exacerbations. Reproduced from [14].

the duration of either symptoms or hospital stay in only 34\%. It also revealed a significant heterogeneity in the definition of outcomes and timing of their evaluation. The DECODE-NET intends to conduct extensive methodological research to address these challenges.

\section{Vision and first steps of the DECODE-NET}

Early career researchers, coupled with more experienced counterparts from over 30 centres from Africa, America, Asia, Europe and Oceania have already joined the DECODE-NET, including respiratory physicians, nurses, physiotherapists, physiologists, trialists, methodologists and statisticians. The network was developed to address the previously described knowledge gaps and methodological challenges, to promote the conduct of high-quality clinical research focusing on RCTs, and ultimately to improve the management and outcomes of COPD exacerbations. The DECODE-NET group envisions development of a global, multidisciplinary, inclusive, independent network that will collectively engage international expertise and resources. Our network strives for excellence in clinical research design and conduct, in making best use of the research data and for promotion of a strong educational ethos to support the development of emerging researchers. The DECODE-NET is an open and collaborative network. Researchers with interest in COPD exacerbations are welcome to join, by contacting any of the authors of this editorial. In parallel, we will seek collaboration with other clinical and research networks working on COPD exacerbations, such as the CICERO (Collaboration In COPD ExaceRbatiOns) ERS Clinical Research Collaboration [28]. CICERO, through the CATALINA observational study, its flagship project, aims to further characterise exacerbations clinically and mechanistically. Innovative findings from the CATALINA study, including much-needed validated diagnostic, prognostic and therapeutic biomarkers, will be fed to the DECODE-NET and are likely to inform future trial design. We believe the two networks complement each other and envisage them to have a close, productive collaboration. DECODE-NET will mainly focus on the conduct of high-quality RCTs, the most rigorous research design that can provide conclusive evidence of the safety and effectiveness of an intervention by minimising potential biases [29]. Pragmatic trial designs, testing the effectiveness of treatments applicable to real-life clinical conditions will be prioritised, to maximise effectiveness [30].

At a recent meeting during the 2019 ERS International Congress we planned several initial research projects that span across the three main research pillars of the network (figure 2): 


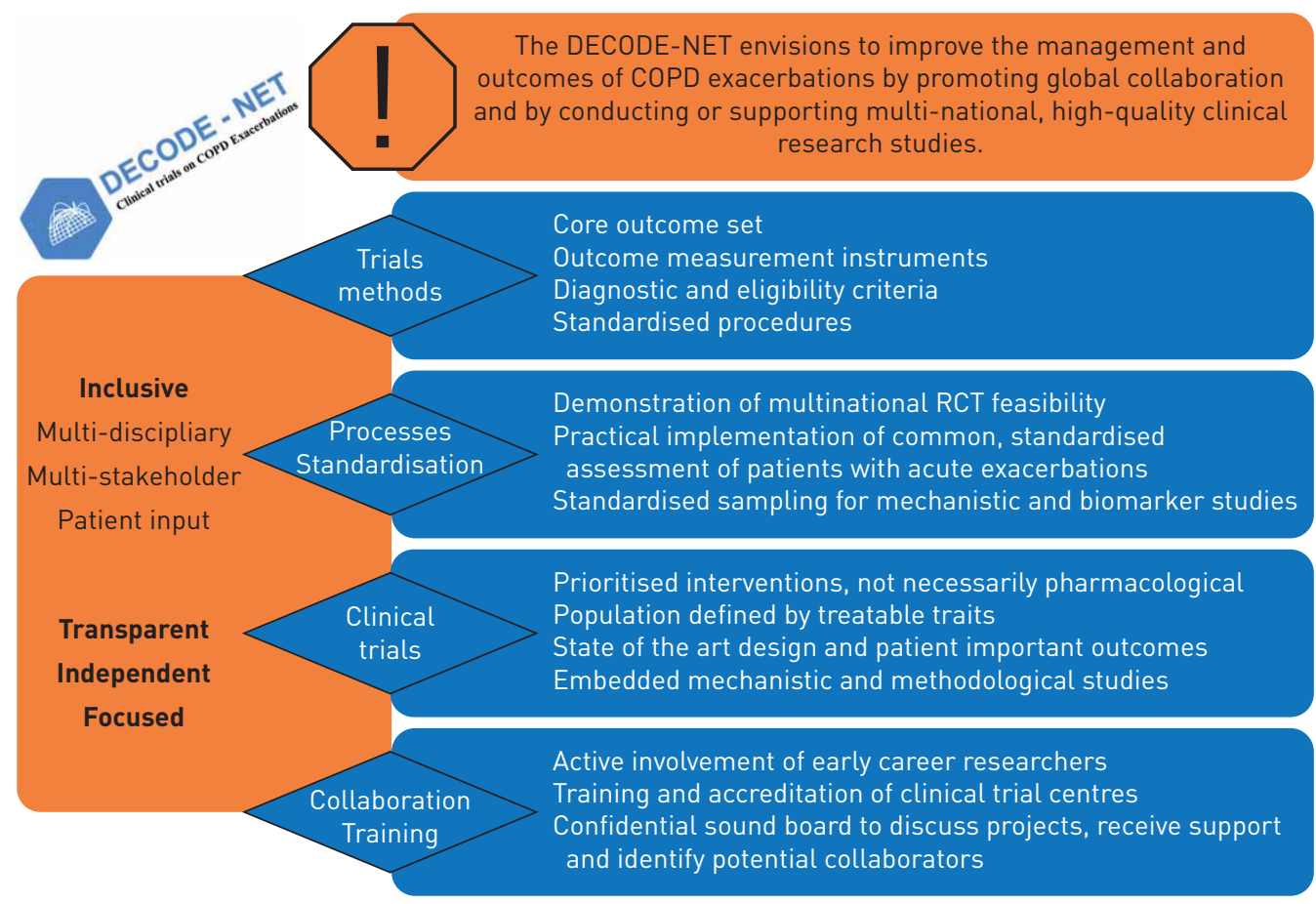

FIGURE 2 The DECODE-NET vision and objectives.

1) Methodological research aiming to improve the quality of RCTs. We will develop a core outcome set for the management of COPD exacerbations. A core outcome set is an agreed minimum set of clinically important outcomes that should be evaluated in all relevant RCTs and could improve their quality and comparability [27]. It is based on international, multi-stakeholder consensus. Members of the network have successfully applied for an ERS task force that is currently undertaking this project. Additional methodological work will focus on the optimisation of the measurement instruments used to assess the outcomes of COPD exacerbations in RCTs.

2) Practical implementation of common and standardised assessment of patients with acute exacerbations of COPD. We will demonstrate that a standardised assessment of the acute patient can be achieved across several centres, thus demonstrating the ability to carry out large-scale high-quality multicentre RCTs with sampling material for mechanistic studies and seek diagnostic, prognostic and therapeutic biomarkers.

3) RCTs aiming to evaluate novel pharmacological and non-pharmacological therapeutic interventions for COPD exacerbations. The main objective of the DECODE-NET is to conduct multinational RCTs. Interventions are to be selected through a prioritisation process involving the network's members and representatives of the patients and other stakeholders. Moreover, DECODE-NET will provide support, methodological and clinical expertise and feedback to any of the members conducting relevant RCTs.

The main initial objective of the DECODE-NET will be to secure additional funding through national or international, public or private funding streams that will allow the conduct of multinational RCTs. Over the past year we found that all DECODE-NET participants face similar challenges and concerns that can indeed be addressed more easily through international collaboration. This resulted in enthusiastic, active involvement of the network's participants that is considered the best guarantee for success.

The DECODE-NET Investigators: Fekri Abroug, Alvar Agusti, Amy Attaway, Per Bakke, Igor Barjaktarevic, Konstantinos Bartziokas, Bianca Beghè, Surya P. Bhatt, Andras Bikov, Thomas Bradbury, Guy Brusselle, Robert Burkes, Cordula Cadus, Marco Contoli, Alexandru Corlateanu, Olga Corlateanu, Gerard Criner, Balazs Csoma, Victoria Dean, Philip Diaz, Mark T. Dransfield, Carlos Echevarria, Josephin Eklöf, Alexander Emelyanov, Rosa Faner, Gustavo Fernandez Romero, Nate Gaeckle, Zeineb Hammouda, MeiLan K. Han, Nicholas S. Hill, Peter Horvath, Arturo Huerta, Anand Iyer, Michael Jacobs, Christine Jenkins, Jens-Ulrik Jensen, Guy Joos, Olga Kharevich, Konstantinos Kostikas, Jerry A. Krishnan, Stephanie LaBedz, David Lafon, Elena Lapteva, Zsofia Lazar, Jörg D. Leuppi, Carol Liddle, John Linnell, Alejandra López-Giraldo, Fernando D. Martinez, Fernando J. Martinez, Alexander G. Mathioudakis, Vanessa McDonald, Takudzwa Mkorombindo, Marilyn Moy, Rune Nielsen, Alberto Papi, Ralph Panos, Trisha Parekh, Ian Pavord, Victor Pinto-Plata, Robert M. Reed, Jean Rommes, Isabel Saraiva, Galina Sergeeva, Frank C. Sciurba, Dave Singh, Pradeesh Sivapalan, Daiana Stolz, Charlie Strange, Hao Wang, Fuqiang Wen, Jørgen Vestbo, James M. Wells, Paula R. Williamson, Janelle Yorke. 
DECODE-NET participating centres: Baystate Health, Springfield, Massachusetts, USA; Belarusian Medical Academy of Postgraduate Education; Birmingham Veterans Affairs Medical Center, Birmingham, Alabama; Cantonal Hospital Basell and Liestal, Basell, Switzerland; Cleveland Clinic, Cleveland, Ohio, USA; Erasmus Medical Centre, Rotterdam, the Netherlands; Ghent University Hospital, Ghent, Belgium; Harvard Medical School, Boston, Massachusetts, USA; Haukeland University Hospital, Bergen, Norway; Herlev-Gentofte Hospital, Hellerup, Denmark; Hospital Clinic, University of Barcelona, Spain; Hospital Fattouma Bourguina, Monastir, Tunisia; John Hunter Hospital, Hunter New England Local Health District, Charlestown, New South Wales, Australia; Lewis Katz School of Medicine, Temple University, Philadelphia, Pennsylvania, USA; Manchester University NHS Foundation Trust, Manchester, UK; Medical University of North Carolina, Chapel Hill, North Carolina, USA; North-Western Medical University, St. Petersburg, Russian Federation; State University of Medicine and Pharmacy "Nicolae Testemitanu", Chisinau, Moldova; Semmelweis University, Budapest, Hungary; The Christie NHS Foundation Trust, Manchester, UK; The George Institute of Health, University of New South Wales, Sydney, Australia; The Ohio State University Wexner Medical Center, Columbus, Ohio, USA; The University of Manchester, Manchester, UK; Tufts Medical Center, Boston, Massachusetts, USA; University Hospital Basel, Basel, Switzerland; University of Alabama at Birmingham, Birmingham, Arizona, USA; University of Ioannina School of Medicine, Ioannina, Greece; University of Basel, Basel, Switzerland; University of Bergen, Bergen, Norway; University of California Los Angeles (UCLA), Los Angeles, California, USA; University of Cincinnati, Cincinnati, Ohio, USA; University of Copenhagen, Denmark; University of Ferrara, Ferrara, Italy; University of Illinois, Chicago, Illinois, USA; University of Liverpool, Liverpool, UK; University of Maryland, Baltimore, Maryland, USA; University of Michigan, Ann Arbor, Michigan, USA; University of Minnesota, Minneapolis, Minnesota, USA; University of Modena and Reggio Emilia, Modena, Italy; University of Newcastle, Callaghan, New South Wales, Australia; University of Pittsburgh, Pittsburgh, Pennsylvania, USA; VA Boston Healthcare System, Boston, Massachusetts, USA; Weil Cornell Medicine, New York, USA; West China Hospital/ West China School of Medicine, Sichuan University, Chengdu, China.

Conflict of interest: A.G. Mathioudakis reports grants from Boehringer Ingelheim, outside the submitted work. P. Sivapalan reports personal fees for lectures from Boehringer Ingelheim, outside the submitted work. A. Papi reports grants, personal fees for advisory board work, consultancy and lectures, and travel expenses reimbursement from GlaxoSmithKline, AstraZeneca, Boehringer Ingelheim, Chiesi Farmaceutici and TEVA, personal fees for advisory board work, consultancy and lectures, and travel expenses reimbursement from Mundipharma, Zambon, Novartis and Sanofi/ Regeneron, grants, personal fees for lectures, and travel expenses reimbursement Menarini, personal fees for advisory board work and consultancy, and travel expenses reimbursement from Roche, grants from Fondazione Maugeri and Fondazione Chiesi, personal fees for consultancy from Edmondpharma, outside the submitted work. J. Vestbo reports personal fees for advisory board work and lectures from AstraZeneca, grants and personal fees for lectures from Boehringer Ingelheim, personal fees for advisory board work and lectures from Chiesi and Novartis, personal fees for advisory board work from GSK, outside the submitted work; and has a family member who is employed by Chiesi.

Support statement: A.G. Mathioudakis and J. Vestbo are supported by the National Institute for Health Research Manchester Biomedical Research Centre (NIHR Manchester BRC). Funding information for this article has been deposited with the Crossref Funder Registry.

\section{References}

1 Wedzicha JA, Miravitlles M, Hurst JR, et al. Management of COPD exacerbations: a European Respiratory Society/American Thoracic Society guideline. Eur Respir J 2017; 49: 1600791.

2 Vogelmeier CF, Criner GJ, Martinez FJ, et al. Global Strategy for the Diagnosis, Management, and Prevention of Chronic Obstructive Lung Disease 2017 Report: GOLD Executive Summary. Eur Respir J 2017; 49: 1700214.

3 Hastie AT, Martinez FJ, Curtis JL, et al. Association of sputum and blood eosinophil concentrations with clinical measures of COPD severity: an analysis of the SPIROMICS cohort. Lancet Respir Med 2017; 5: 956-967.

4 Gayle A, Dickinson S, Morris K, et al. What is the impact of GOLD 2017 recommendations in primary care? - a descriptive study of patient classifications, treatment burden and costs. Int J Chron Obstruct Pulmon Dis 2018; 13: 3485-3492.

5 Kunisaki KM, Dransfield MT, Anderson JA, et al. Exacerbations of chronic obstructive pulmonary disease and cardiac events. A post hoc cohort analysis from the SUMMIT randomized clinical trial. Am J Respir Crit Care Med 2018; 198: 51-57.

6 Wedzicha JA, Seemungal TA. COPD exacerbations: defining their cause and prevention. Lancet 2007; 370: 786-796.

7 Zhang Y, Morgan RL, Alonso-Coello P, et al. A systematic review of how patients value COPD outcomes. Eur Respir J 2018; 52: 1800222.

8 Rodriguez-Roisin R. Toward a consensus definition for COPD exacerbations. Chest 2000; 117: 398S-401S.

9 Mathioudakis AG, Janner J, Moberg M, et al. A systematic evaluation of the diagnostic criteria for COPD and exacerbations used in randomised controlled trials on the management of COPD exacerbations. ERJ Open Res 2019; 5: 00136-2019.

10 Kim V, Aaron SD. What is a COPD exacerbation? Current definitions, pitfalls, challenges and opportunities for improvement. Eur Respir J 2018; 52: 1801262.

11 Scioscia G, Blanco I, Arismendi E, et al. Different dyspnoea perception in COPD patients with frequent and infrequent exacerbations. Thorax 2017; 72: 117-121.

12 Beghe B, Verduri A, Roca M, et al. Exacerbation of respiratory symptoms in COPD patients may not be exacerbations of COPD. Eur Respir J 2013; 41: 993-995.

13 Adamson PD, Anderson JA, Brook RD, et al. Cardiac troponin I and cardiovascular risk in patients with chronic obstructive pulmonary disease. J Am Coll Cardiol 2018; 72: 1126-1137.

14 Mathioudakis AG, Janssens W, Sivapalan P, et al. Acute exacerbations of chronic obstructive pulmonary disease: In search of diagnostic biomarkers and treatable traits. Thorax 2020; 75: 520-527.

15 Walters MI, Edwards PR, Waterhouse JC, et al. Long term domiciliary oxygen therapy in chronic obstructive pulmonary disease. Thorax 1993; 48: 1170-1177. 
16 Vollenweider DJ, Frei A, Steurer-Stey CA, et al. Antibiotics for exacerbations of chronic obstructive pulmonary disease. Cochrane Database Syst Rev 2018; 10: CD010257.

17 Mathioudakis AG, Chatzimavridou-Grigoriadou V, Corlateanu A, et al. Procalcitonin to guide antibiotic administration in COPD exacerbations: a meta-analysis. Eur Respir Rev 2017; 26: 160073.

18 Sivapalan P, Lapperre TS, Janner J, et al. Eosinophil-guided corticosteroid therapy in patients admitted to hospital with COPD exacerbation (CORTICO-COP): a multicentre, randomised, controlled, open-label, non-inferiority trial. Lancet Respir Med 2019; 7: 699-709.

19 Bafadhel M, McKenna S, Terry S, et al. Blood eosinophils to direct corticosteroid treatment of exacerbations of chronic obstructive pulmonary disease: a randomized placebo-controlled trial. Am J Respir Crit Care Med 2012; 186: $48-55$.

20 McDonald VM, Osadnik CR, Gibson PG. Treatable traits in acute exacerbations of chronic airway diseases. Chron Respir Dis 2019; 16: 1479973119867954.

21 Nottage KA, Hankins JS, Faughnan LG, et al. Addressing challenges of clinical trials in acute pain: The Pain Management of Vaso-occlusive Crisis in Children and Young Adults with Sickle Cell Disease Study. Clin Trials 2016; 13: 409-416.

22 Holcomb JB, Weiskopf $\mathrm{R}$, Champion $\mathrm{H}$, et al. Challenges to effective research in acute trauma resuscitation: consent and endpoints. Shock 2011; 35: 107-113.

23 Johnson AM, Jones SB, Duncan PW, et al. Hospital recruitment for a pragmatic cluster-randomized clinical trial: Lessons learned from the COMPASS study. Trials 2018; 19: 74.

24 van Staa TP, Dyson L, McCann G, et al. The opportunities and challenges of pragmatic point-of-care randomised trials using routinely collected electronic records: evaluations of two exemplar trials. Health Technol Assess 2014; 18: $1-146$.

25 Woodcock J, LaVange LM. Master protocols to study multiple therapies, multiple diseases, or both. N Engl J Med 2017; 377: 62-70.

26 Williamson PR, Altman DG, Bagley H, et al. The COMET Handbook: version 1.0. Trials 2017; 18: 280

27 Mathioudakis AG, Moberg M, Janner J, et al. Outcomes reported on the management of COPD exacerbations: a systematic survey of randomised controlled trials. ERJ Open Res 2019; 5: 00072-2019.

28 Janssens W, Bafadhel M. The CICERO (Collaboration In COPD ExaceRbatiOns) Clinical Research Collaboration. Eur Respir J 2020; 55: 2000079.

29 Collins R, Bowman L, Landray M, et al. The magic of randomization versus the myth of real-world evidence. $N$ Engl J Med 2020; 382: 674-678.

30 Woodcock A, Boucot I, Leather DA, et al. Effectiveness versus efficacy trials in COPD: how study design influences outcomes and applicability. Eur Respir J 2018; 51: 1701531. 